\title{
The Asymptotic Behavior of a Stochastic Predator-Prey System with Holling II Functional Response
}

\author{
Zhenwen Liu, ${ }^{1,2}$ Ningzhong Shi, ${ }^{1}$ Daqing Jiang, ${ }^{1}$ \\ and Chunyan $\mathrm{Ji}^{1}, 3$ \\ ${ }^{1}$ School of Mathematics and Statistics, Northeast Normal University, Changchun, Jilin 130024, China \\ ${ }^{2}$ School of Science, Changchun University of Science and Technology, Changchun, Jilin 130022, China \\ ${ }^{3}$ Department of Mathematics, Changshu Institute of Technology, Changshu, Jiangsu 215500, China
}

Correspondence should be addressed to Daqing Jiang, daqingjiang2010@hotmail.com

Received 21 October 2012; Accepted 14 December 2012

Academic Editor: Ivanka Stamova

Copyright (c) 2012 Zhenwen Liu et al. This is an open access article distributed under the Creative Commons Attribution License, which permits unrestricted use, distribution, and reproduction in any medium, provided the original work is properly cited.

We discuss a stochastic predator-prey system with Holling II functional response. First, we show that this system has a unique positive solution as this is essential in any population dynamics model. Then, we deduce the conditions that there is a stationary distribution of the system, which implies that the system is permanent. At last, we give the conditions for the system that is going to be extinct.

\section{Introduction}

One of the most popular predator-prey model is the one with Michaelis-Menten type (or Holling Type II) functional response [1, 2]:

$$
\begin{gathered}
\dot{x}(t)=x(t)\left(a-b x(t)-\frac{\alpha y(t)}{1+\beta x(t)}\right), \\
\dot{y}(t)=y(t)\left(-e+\frac{k \alpha x(t)}{1+\beta x(t)}\right),
\end{gathered}
$$

where $x(t)$ and $y(t)$ are the population densities of prey and predator at time $t$, respectively. The constants $a, b / a, \alpha, \beta, e$, and $k$ are positive constants that stand for prey intrinsic growth rate, carrying capacity, the maximum ingestion rate, half-saturation constant, predator death rate, and the conversion factor, respectively. This model exhibits the well-known but highly 
controversial "paradox of enrichment" observed by Hairston et al. [3] and by Rosenzweig [4] which is rarely reported in nature. It is very important to study the existence and asymptotical stability of equilibria and limit cycle for autonomous predator-prey systems with Holling II functional response. If $k a \alpha \beta>a e \beta^{2}+k b \alpha+b e \beta$, then system (1.1) has a unique limit cycle which is stable. If $a k \alpha>a e \beta+b e$, then system (1.1) has a unique positive equilibrium:

$$
x^{*}=\frac{e}{k \alpha-e \beta^{\prime}}, \quad y^{*}=\frac{k \alpha(a k \alpha-a e \beta-b e)}{(k \alpha-e \beta)^{2}},
$$

which is a stable node or focus (see [5]).

However, countless organisms live in seasonally or diurnally forced environments. Hence, authors considered models with periodic ecological parameters or perturbations. For example, Liu and Chen [6] introduced periodic constant impulsive immigration of predator into system (1.1) and gave conditions for the system to be extinct and permanence, respectively. Zhang and Chen [7] studied a Holling II functional response food chain model with impulsive perturbations. Zhang et al. [8] further considered system (1.1) with periodic constant impulsive immigration of predator and periodic variation in the intrinsic growth rate of the prey.

On the other hand, the white noise is always present, and we cannot omit the influence of the white noise to the system. May [9] pointed out that due to continuous fluctuation in the environment, the birth rates, death rates, carrying capacity, competition coefficients, and all other parameters involved with the model exhibit random fluctuation to a great lesser extent, and as a result the equilibrium population distribution never attains a steady value, but fluctuates randomly around some average value. Many authors studied the effect of the stochastic perturbation to the predator-prey system with different functional responses, such as [10-14]. Therefore, in this paper, we also introduce stochastic perturbation system (1.1) and obtain the following stochastic system:

$$
\begin{gathered}
d x(t)=x(t)\left(a-b x(t)-\frac{\alpha y(t)}{1+\beta x(t)}\right) d t+\sigma_{1} x(t) d B_{1}(t), \\
d y(t)=y(t)\left(-e+\frac{k \alpha x(t)}{1+\beta x(t)}\right) d t+\sigma_{2} y(t) d B_{2}(t)
\end{gathered}
$$

where $B_{1}(t)$ and $B_{2}(t)$ are mutually independent Brownian motion with $B_{1}(0)=B_{2}(0)=0$, and $\sigma_{1}^{2}, \sigma_{2}^{2}$ are intensities of the white noise.

The aim of this paper is to discuss the long time behavior of system (1.3). As the deterministic population models, we are also interested in the permanence and extinction of the system. The global stability of the positive equilibrium means that the system is permanence. But, for the stochastic system, there is no positive equilibrium. Hence, it is impossible that the solution of system (1.3) will tend to a fixed point. In this paper, we show that there is a stationary distribution of system (1.3) mainly according to the theory of Has'meminskii [15], if the white noise is small. While if the white noise is large, based on the techniques developed in $[16,17]$, we prove that the predator population will die out a.s. and the prey population will either extinct or its distribution converges to a probability measure. It does not happen that both the prey population and the predator population in system (1.3) will die out, which is brought by large white noise, such as weather, epidemic 
disease. From this point, we say the stochastic model is more realistic than the deterministic model.

The rest of this paper is organized as follows. In Section 2, we show that there is a unique nonnegative solution of system (1.3). In Section 3, we show that there is a stationary distribution under small white noise. While in Section 4, we consider the situation when the white noise is large. We prove that the system will be extinct. Finally, we give an appendix containing the stationary distribution theory used in Section 3.

\section{Existence and Uniqueness of the Nonnegative Solution}

To investigate the dynamical behavior, the first concern is the global existence of the solutions. Hence in this section we show that the solution of system (1.3) is global and nonnegative. It is not difficult to check the uniqueness and global existence of solutions if the coefficients of the equation satisfy the linear growth condition and local Lipschitz condition (cf. [18]). However, the coefficients of system (1.3) do not satisfy the linear growth condition, but locally Lipschitz continuous, so the solution of system (1.3) may explode at a finite time. In this section, by changing variables, we first show that system (1.3) has a local solution, then show that this solution is global.

Theorem 2.1. For any initial value $(x(0), y(0)) \in R_{+}^{2}$, there is a unique solution $(x(t), y(t))$ of system (1.3) on $t \geq 0$, and the solution will remain in $R_{+}^{2}$ with probability 1.

Proof. First, consider the following system, by changing variables, $x(t)=e^{u(t)}, y(t)=e^{v(t)}$,

$$
\begin{gathered}
d u(t)=\left(a-\frac{\sigma_{1}^{2}}{2}-b e^{u(t)}-\frac{\alpha e^{v(t)}}{1+\beta e^{u(t)}}\right) d t+\sigma_{1} d B_{1}(t), \\
d v(t)=\left(-e-\frac{\sigma_{2}^{2}}{2}+\frac{k \alpha e^{u(t)}}{1+\beta e^{u(t)}}\right) d t+\sigma_{2} d B_{2}(t) .
\end{gathered}
$$

It is clear that the coefficients of system (2.1) are locally Lipschitz continuous for the given initial value $(\log x(0), \log y(0)) \in R^{2}$ there is a unique local solution $(u(t), v(t))$ on $t \in\left[0, \tau_{e}\right)$, where $\tau_{e}$ is the explosion time (see [18]). Hence, by Itô formula, we know $\left(e^{u(t)}, e^{v(t)}\right), t \in$ $\left[0, \tau_{e}\right)$ is a unique positive local solution of system (1.3). To show that this solution is global, we need to show that $\tau_{e}=\infty$ a.s. Let $m_{0} \geq 1$ be sufficiently large so that $x(0), y(0)$ all lie within the interval $\left[1 / m_{0}, m_{0}\right]$. For each integer $m \geq m_{0}$, define the stopping time:

$$
\tau_{m}=\inf \left\{t \in\left[0, \tau_{e}\right): \min \{x(t), y(t)\} \leq \frac{1}{m} \text { or } \max \{x(t), y(t)\} \geq m\right\}
$$

Where, throughout this paper, we set $\inf \emptyset=\infty$ (as usual $\emptyset$ denotes the empty set). Clearly, $\tau_{m}$ is increasing as $m \rightarrow \infty$. Set $\tau_{\infty}=\lim _{m \rightarrow \infty} \tau_{m}$, whence $\tau_{\infty} \leq \tau_{e}$ a.s. If we can show that $\tau_{\infty}=\infty$ a.s., then $\tau_{e}=\infty$ and $(x(t), y(t)) \in R_{+}^{2}$ a.s. for all $t \geq 0$. In other words, to complete the proof all we need to show is that $\tau_{\infty}=\infty$ a.s. If this statement is false, then there is a pair of constants $T>0$ and $\epsilon \in(0,1)$ such that

$$
P\left\{\tau_{\infty} \leq T\right\}>\epsilon .
$$


Hence there is an integer $m_{1} \geq m_{0}$ such that

$$
P\left\{\tau_{m} \leq T\right\} \geq \epsilon \quad \forall m \geq m_{1} .
$$

Define a $C^{2}$-function $V: R_{+}^{2} \rightarrow \bar{R}_{+}$by

$$
V(x, y)=\left(x-c-c \log \frac{x}{c}\right)+\frac{1}{k}(y-1-\log y)
$$

where $c$ is a positive constant to be determined later. The nonnegativity of this function can be seen from $u-1-\log u \geq 0$, for all $u>0$. Using Itô's formula, we get

$$
d V:=L V d t+\sigma_{1}(x-c) d B_{1}(t)+\frac{\sigma_{2}}{k}(y-1) d B_{2}(t),
$$

where

$$
\begin{aligned}
L V & =(x-c)\left(a-b x-\frac{\alpha y}{1+\beta x}\right)+\frac{c \sigma_{1}^{2}}{2}+\frac{1}{k}(y-1)\left(-e+\frac{k \alpha y}{1+\beta x}\right)+\frac{\sigma_{2}^{2}}{2 k} \\
& =-a c+\frac{c \sigma_{1}^{2}}{2}+\frac{e}{k}+\frac{\sigma_{2}^{2}}{2 k}+(a+b c) x-\frac{e}{k} y-b x^{2}+\frac{\alpha c y}{1+\beta x} \\
& \leq-a c+\frac{c \sigma_{1}^{2}}{2}+\frac{e}{k}+\frac{\sigma_{2}^{2}}{2 k}+(a+b c) x-b x^{2}-\left(\frac{e}{k}-\alpha c\right) y .
\end{aligned}
$$

Choose $c=e / \alpha k$ such that $e / k-\alpha c=0$, then

$$
L V \leq-a c+\frac{c \sigma_{1}^{2}}{2}+\frac{e}{k}+\frac{\sigma_{2}^{2}}{2 k}+(a+b c) x-b x^{2} \leq K
$$

where $K$ is a positive constant. Therefore

$$
\begin{aligned}
\int_{0}^{\tau_{m} \wedge T} d V(x(t), y(t)) \leq & \int_{0}^{\tau_{m} \wedge T} K d t \\
& +\int_{0}^{\tau_{m} \wedge T} \sigma_{1}(x(s)-c) d B_{1}(s)+\frac{\sigma_{2}}{k}(y(s)-1) d B_{2}(s),
\end{aligned}
$$

which implies that,

$$
E\left[V\left(x\left(\tau_{m} \wedge T\right), y\left(\tau_{m} \wedge T\right)\right)\right] \leq V(x(0), y(0))+E \int_{0}^{\tau_{m} \wedge T} K d t \leq V(x(0), y(0))+K T .
$$


Set $\Omega_{m}=\left\{\tau_{m} \leq T\right\}$ for $m \geq m_{1}$, then by (2.4), we know that $P\left(\Omega_{m}\right) \geq \epsilon$. Note that for every $\omega \in \Omega_{m}$, there is at least one of $x\left(\tau_{m}, \omega\right)$ and $y\left(\tau_{m}, \omega\right)$ equals either $m$ or $1 / m$, then

$$
\begin{aligned}
V\left(x\left(\tau_{m}\right), y\left(\tau_{m}\right)\right) \geq & \left(m-c-c \log \frac{m}{c}\right) \wedge\left(\frac{1}{m}-c+c \log (c m)\right) \\
& \wedge \frac{1}{k}(m-1-\log m) \wedge \frac{1}{k}\left(\frac{1}{m}-1+\log m\right) .
\end{aligned}
$$

It then follows from (2.4) and (2.10) that

$$
\begin{aligned}
V(x(0), y(0))+K T \geq & E\left[1_{\Omega_{m}(w)} V\left(x\left(\tau_{m}\right), y\left(\tau_{m}\right)\right)\right] \\
\geq & \epsilon\left(m-c-c \log \frac{m}{c}\right) \wedge\left(\frac{1}{m}-c+c \log (c m)\right) \wedge \frac{1}{k}(m-1-\log m) \\
& \wedge \frac{1}{k}\left(\frac{1}{m}-1+\log m\right),
\end{aligned}
$$

where $1_{\Omega_{m}(\omega)}$ is the indicator function of $\Omega_{m}$. Letting $m \rightarrow \infty$ leads to the contradiction that $\infty>V(x(0), y(0))+K T=\infty$. So we must therefore have $\tau_{\infty}=\infty$ a.s.

\section{Permanence}

There is no equilibrium of system (1.3). Hence we cannot show the permanence of the system by proving the stability of the positive equilibrium as the deterministic system. In this section we show that there is a stationary distribution of system (1.3).

Remark 3.1. Theorem 2.1 shows that there exists a unique positive solution $(x(t), y(t))$ of system (1.3) with any initial value $(x(0), y(0)) \in R_{+}^{2}$. From the proof of Theorem 2.1, we obtain that $L V \leq K$. Define $\tilde{V}=V+K$, then $L \tilde{V} \leq \tilde{V}$, and it is clear that $\tilde{V}_{R}=$ $\inf _{(x, y) \in R_{+}^{2} \backslash D_{k}} \tilde{V}(x, y) \rightarrow \infty$ as $k \rightarrow \infty$, where $D_{k}=(1 / k, k) \times(1 / k, k)$. Hence by Remark 2 of Theorem 4.1 of Has'meminskii, 1980, page 86 in [15], we obtain that the solution $(x(t), y(t))$ is a homogeneous Markov process in $R_{+}^{2}$.

Theorem 3.2. If $a e \beta+b e<a k \alpha<a e \beta+b k \alpha / \beta$ and $\sigma_{1}>0, \sigma_{2}>0$ such that $\sigma_{2}^{2}<k \alpha x^{*} /\left(1+\beta x^{*}\right)$ and

$$
\begin{aligned}
\left(\frac{1}{2}+\right. & \left.l_{2} x^{*}\right) x^{*} \sigma_{1}^{2}+\left(\frac{1+\beta x^{*}}{2}+\frac{l_{2} y^{*}}{k}\right) \frac{y^{*} \sigma_{2}^{2}}{k} \\
& <\min \left\{\frac{1}{2}\left(b-\frac{\alpha \beta y^{*}}{1+\beta x^{*}}\right)\left(x^{*}\right)^{2}, \frac{l_{2}}{2}\left(\frac{\alpha x^{*}}{k\left(1+\beta x^{*}\right)}-\frac{\sigma_{2}^{2}}{k^{2}}\right)\left(y^{*}\right)^{2}\right\},
\end{aligned}
$$

where $\left(x^{*}, y^{*}\right)$ is the positive equilibrium of system (1.1) and $l_{2}$ is defined as in the proof. Then system (1.3) has a stationary ergodic solution. 
Proof. Since $a k \alpha>a e \beta+b e$, then there is a positive equilibrium $\left(x^{*}, y^{*}\right)$ of system (1.1), and

$$
a=b x^{*}+\frac{\alpha y^{*}}{1+\beta x^{*}}, \quad e=\frac{k \alpha x^{*}}{1+\beta x^{*}} .
$$

Let

$$
V_{1}(x, y)=\left(x-x^{*}-x^{*} \log \frac{x}{x^{*}}\right)+l_{1}\left(y-y^{*}-y^{*} \log \frac{y}{y^{*}}\right)
$$

where $l_{1}$ is a positive constant to be determined later. Let $L$ be the generating operator of system (1.3). Then

$$
\begin{aligned}
L V_{1}= & \left(x-x^{*}\right)\left(a-b x-\frac{\alpha y}{1+\beta x}\right)+\frac{x^{*} \sigma_{1}^{2}}{2}+l_{1}\left(y-y^{*}\right)\left(-e+\frac{k \alpha x}{1+\beta x}\right)+\frac{l_{1} y^{*} \sigma_{2}^{2}}{2} \\
= & \left(x-x^{*}\right)\left[-b\left(x-x^{*}\right)-\frac{\alpha}{1+\beta x}\left(y-y^{*}\right)+\frac{\alpha \beta y^{*}}{\left(1+\beta x^{*}\right)(1+\beta x)}\left(x-x^{*}\right)\right]+\frac{x^{*} \sigma_{1}^{2}}{2} \\
& +l_{1}\left(y-y^{*}\right) \frac{k \alpha\left(x-x^{*}\right)}{\left(1+\beta x^{*}\right)(1+\beta x)}+\frac{l_{1} y^{*} \sigma_{2}^{2}}{2} \\
\leq & -\left(b-\frac{\alpha \beta y^{*}}{1+\beta x^{*}}\right)\left(x-x^{*}\right)^{2}-\frac{\alpha}{1+\beta x}\left(1-\frac{l_{1} k}{1+\beta x^{*}}\right)\left(x-x^{*}\right)\left(y-y^{*}\right)+\frac{x^{*} \sigma_{1}^{2}}{2}+\frac{l_{1} y^{*} \sigma_{2}^{2}}{2}
\end{aligned}
$$

Choose $l_{1}=\left(1+\beta x^{*}\right) / k$ such that $1-l_{1} k /\left(1+\beta x^{*}\right)=0$ and yields

$$
L V_{1} \leq-\left(b-\frac{\alpha \beta y^{*}}{1+\beta x^{*}}\right)\left(x-x^{*}\right)^{2}+\frac{x^{*} \sigma_{1}^{2}}{2}+\frac{\left(1+\beta x^{*}\right) y^{*} \sigma_{2}^{2}}{2 k}
$$

Let

$$
V_{2}(x, y)=\frac{1}{2}\left[\left(x-x^{*}\right)+\frac{1}{k}\left(y-y^{*}\right)\right]^{2}
$$

Note that

$$
\begin{aligned}
d\left[\left(x-x^{*}\right)+\frac{1}{k}\left(y-y^{*}\right)\right]= & \left(a x-b x^{2}-\frac{e}{k} y\right) d t+\sigma_{1} x d B_{1}(t)+\frac{\sigma_{2}}{k} y d B_{2}(t) \\
= & {\left[-b x\left(x-x^{*}\right)^{2}+\alpha \frac{y^{*}\left(x-x^{*}\right)-x^{*}\left(y-y^{*}\right)}{1+\beta x^{*}}\right] d t } \\
& +\sigma_{1} x d B_{1}(t)+\frac{\sigma_{2}}{k} y d B_{2}(t)
\end{aligned}
$$


then

$$
\begin{aligned}
L V_{2}= & {\left[\left(x-x^{*}\right)+\frac{1}{k}\left(y-y^{*}\right)\right]\left[-b x\left(x-x^{*}\right)^{2}+\alpha \frac{y^{*}\left(x-x^{*}\right)-x^{*}\left(y-y^{*}\right)}{1+\beta x^{*}}\right]+\frac{\sigma_{1}^{2}}{2} x^{2}+\frac{\sigma_{2}^{2}}{2 k^{2}} y^{2} } \\
= & -b x\left(x-x^{*}\right)^{2}+\left(\frac{\alpha y^{*}}{1+\beta x^{*}}+\frac{b y^{*}}{k}\right)\left(x-x^{*}\right)^{2}-\frac{b}{k}\left(x-x^{*}\right)^{2} y-\frac{\alpha x^{*}}{k\left(1+\beta x^{*}\right)}\left(y-y^{*}\right)^{2} \\
& +\left(\frac{\alpha y^{*}}{k\left(1+\beta x^{*}\right)}-\frac{\alpha x^{*}}{1+\beta x^{*}}-\frac{b x^{*}}{k}\right)\left(x-x^{*}\right)\left(y-y^{*}\right)+\frac{\sigma_{1}^{2}}{2} x^{2}+\frac{\sigma_{2}^{2}}{2 k^{2}} y^{2} \\
\leq & \left(\frac{\alpha y^{*}}{1+\beta x^{*}}+\frac{b y^{*}}{k}+\sigma_{1}^{2}\right)\left(x-x^{*}\right)^{2}-\left(\frac{\alpha x^{*}}{k\left(1+\beta x^{*}\right)}-\frac{\sigma_{2}^{2}}{k^{2}}\right)\left(y-y^{*}\right)^{2} \\
& +\left(\frac{\alpha y^{*}}{k\left(1+\beta x^{*}\right)}-\frac{\alpha x^{*}}{1+\beta x^{*}}-\frac{b x^{*}}{k}\right)\left(x-x^{*}\right)\left(y-y^{*}\right)+\sigma_{1}^{2}\left(x^{*}\right)^{2}+\frac{\sigma_{2}^{2}}{k^{2}}\left(y^{*}\right)^{2},
\end{aligned}
$$

where $L$ is also the generating operator of system (1.3). Note that

$$
\begin{aligned}
\left(\frac{\alpha y^{*}}{k\left(1+\beta x^{*}\right)}-\frac{\alpha x^{*}}{1+\beta x^{*}}-\frac{b x^{*}}{k}\right)\left(x-x^{*}\right)\left(y-y^{*}\right) & \\
\leq & \frac{\left(\alpha y^{*} /\left(k\left(1+\beta x^{*}\right)\right)-\alpha x^{*} /\left(1+\beta x^{*}\right)-b x^{*} / k\right)^{2}}{2\left(\alpha x^{*} /\left(k\left(1+\beta x^{*}\right)\right)-\sigma_{2}^{2} / k^{2}\right)}\left(x-x^{*}\right)^{2} \\
& +\frac{1}{2}\left(\frac{\alpha x^{*}}{k\left(1+\beta x^{*}\right)}-\frac{\sigma_{2}^{2}}{k^{2}}\right)\left(y-y^{*}\right)^{2} \\
:= & \delta\left(x-x^{*}\right)^{2}+\frac{1}{2}\left(\frac{\alpha x^{*}}{k\left(1+\beta x^{*}\right)}-\frac{\sigma_{2}^{2}}{k^{2}}\right)\left(y-y^{*}\right)^{2}
\end{aligned}
$$

then

$$
\begin{aligned}
L V_{2} \leq & \left(\frac{\alpha y^{*}}{1+\beta x^{*}}+\frac{b y^{*}}{k}+\sigma_{1}^{2}+\delta\right)\left(x-x^{*}\right)^{2}-\frac{1}{2}\left(\frac{\alpha x^{*}}{k\left(1+\beta x^{*}\right)}-\frac{\sigma_{2}^{2}}{k^{2}}\right)\left(y-y^{*}\right)^{2} \\
& +\sigma_{1}^{2}\left(x^{*}\right)^{2}+\frac{\sigma_{2}^{2}}{k^{2}}\left(y^{*}\right)^{2} .
\end{aligned}
$$

Now define

$$
V(x, y)=V_{1}(x, y)+l_{2} V_{2}(x, y)
$$


where $l_{2}$ is a positive constant to be determined later. Then

$$
\begin{aligned}
L V \leq & -\left(b-\frac{\alpha \beta y^{*}}{1+\beta x^{*}}-l_{2}\left(\frac{\alpha y^{*}}{1+\beta x^{*}}+\frac{b y^{*}}{k}+\sigma_{1}^{2}+\delta\right)\right)\left(x-x^{*}\right)^{2} \\
& -\frac{l_{2}}{2}\left(\frac{\alpha x^{*}}{k\left(1+\beta x^{*}\right)}-\frac{\sigma_{2}^{2}}{k^{2}}\right)\left(y-y^{*}\right)^{2}+\left(\frac{1}{2}+l_{2} x^{*}\right) x^{*} \sigma_{1}^{2}+\left(\frac{1+\beta x^{*}}{2}+\frac{l_{2} y^{*}}{k}\right) \frac{y^{*} \sigma_{2}^{2}}{k} .
\end{aligned}
$$

Choose $l_{2}>0$ such that $\left(b-\alpha \beta y^{*} /\left(1+\beta x^{*}\right)-l_{2}\left(\alpha y^{*} /\left(1+\beta x^{*}\right)+b y^{*} / k+\sigma_{1}^{2}+\delta\right)\right)=(1 / 2)(b-$ $\left.\alpha \beta y^{*} /\left(1+\beta x^{*}\right)\right)$, then it follows from (3.12) that

$$
\begin{aligned}
L V \leq & -\frac{1}{2}\left(b-\frac{\alpha \beta y^{*}}{1+\beta x^{*}}\right)\left(x-x^{*}\right)^{2}-\frac{l_{2}}{2}\left(\frac{\alpha x^{*}}{k\left(1+\beta x^{*}\right)}-\frac{\sigma_{2}^{2}}{k^{2}}\right)\left(y-y^{*}\right)^{2} \\
& +\left(\frac{1}{2}+l_{2} x^{*}\right) x^{*} \sigma_{1}^{2}+\left(\frac{1+\beta x^{*}}{2}+\frac{l_{2} y^{*}}{k}\right) \frac{y^{*} \sigma_{2}^{2}}{k} .
\end{aligned}
$$

Note that

$$
\begin{aligned}
\left(\frac{1}{2}+\right. & \left.l_{2} x^{*}\right) x^{*} \sigma_{1}^{2}+\left(\frac{1+\beta x^{*}}{2}+\frac{l_{2} y^{*}}{k}\right) \frac{y^{*} \sigma_{2}^{2}}{k} \\
& <\min \left\{\frac{1}{2}\left(b-\frac{\alpha \beta y^{*}}{1+\beta x^{*}}\right)\left(x^{*}\right)^{2}, \frac{l_{2}}{2}\left(\frac{\alpha x^{*}}{k\left(1+\beta x^{*}\right)}-\frac{\sigma_{2}^{2}}{k^{2}}\right)\left(y^{*}\right)^{2}\right\}
\end{aligned}
$$

then the ellipsoid

$$
\begin{gathered}
-\frac{1}{2}\left(b-\frac{\alpha \beta y^{*}}{1+\beta x^{*}}\right)\left(x-x^{*}\right)^{2}-\frac{l_{2}}{2}\left(\frac{\alpha x^{*}}{k\left(1+\beta x^{*}\right)}-\frac{\sigma_{2}^{2}}{k^{2}}\right)\left(y-y^{*}\right)^{2} \\
+\left(\frac{1}{2}+l_{2} x^{*}\right) x^{*} \sigma_{1}^{2}+\left(\frac{1+\beta x^{*}}{2}+\frac{l_{2} y^{*}}{k}\right) \frac{y^{*} \sigma_{2}^{2}}{k}=0
\end{gathered}
$$

lies entirely in $R_{+}^{2}$. We can take $U$ to be a neighborhood of the ellipsoid with $\bar{U} \subseteq E_{l}=R_{+}^{2}$, so that for $(x, y) \in U \backslash E_{l}, L V \leq-C$ (C is a positive constant), which implies condition (B.2) in Lemma A.1 is satisfied. Hence the solution $(x(t), y(t))$ is recurrent in the domain $U$, which together with Lemma A.3 and Remark 3.1 implies that $(x(t), y(t))$ is recurrent in any bounded domain $D \subset R_{+}^{2}$. Besides, for all $D$, there is an $M=\min \left\{\sigma_{1}^{2} x^{2}, \sigma_{2}^{2} y^{2},(x, y) \in \bar{D}\right\}>0$ such that

$$
\sum_{i, j=1}^{2} \lambda_{i j} \xi_{i} \xi_{j}=\sigma_{1}^{2} x^{2} \xi_{1}^{2}+\sigma_{2}^{2} y^{2} \xi_{2}^{2} \geq M\left|\xi^{2}\right| \quad \text { all } x \in \bar{D}, \xi \in R^{2}
$$

which implies that condition (B.1) is also satisfied. Therefore, system (1.3) has a stable a stationary distribution $\mu(\cdot)$ and it is ergodic. 
Note that

$$
\begin{aligned}
d x^{p} & =p x^{p}\left(a-b x-\frac{\alpha y}{1+\beta x}\right) d t+p \sigma_{1} x^{p} d B_{1}(t)+\frac{1}{2} p(p-1) \sigma_{1}^{2} x^{p} d t \\
& \leq p x^{p}\left(a+\frac{p}{2} \sigma_{1}^{2}-b x\right) d t+p \sigma_{1} x^{p} d B_{1}(t)
\end{aligned}
$$

then

$$
\begin{aligned}
\frac{d E\left[x^{p}\right]}{d t} & \leq p\left(a+\frac{p \sigma_{1}^{2}}{2}\right) E\left[x^{p}\right]-b E\left[x^{p+1}\right] \\
& \leq p\left(a+\frac{p \sigma_{1}^{2}}{2}\right) E\left[x^{p}\right]-b\left(E\left[x^{p}\right]\right)^{(p+1) / p}
\end{aligned}
$$

Hence by comparison theorem, we get

$$
\limsup _{t \rightarrow \infty} E\left[x^{p}(t)\right] \leq\left(\frac{a+p \sigma_{1}^{2} / 2}{b}\right)^{p}
$$

by which together with the continuity of $E\left[x^{p}(t)\right]$, we have that there exists a positive constant $K=K(p)$ such that

$$
E\left[x^{p}(t)\right] \leq K(p)
$$

By Doob's martingale inequality, together with the (3.20), for $\delta>0$, we have

$$
P\left\{\omega: \sup _{(n-1) \delta \leq t \leq n \delta} \frac{x(t)}{t}>\delta\right\} \leq \frac{E\left[x^{p}(n \delta)\right]}{(n \delta)^{p} \delta} \leq \frac{K(p)}{n^{p} \delta^{p+1}}, \quad p>1 .
$$

In view of the well-known Borel-Cantelli lemma, we see that for almost all $\omega \in \Omega$,

$$
\sup _{(n-1) \delta \leq t \leq n \delta} \frac{x(t)}{t} \leq \delta
$$

holds for all but finitely many $n$. Hence there exists an $n_{0}(\omega)$, for all $\omega \in \Omega$ excluding a $P$-null set, for which (3.22) holds whenever $n \geq n_{0}$. Consequently, letting $\delta \rightarrow 0$, we have, for almost all $\omega$,

$$
\lim _{t \rightarrow \infty} \frac{x(t)}{t}=0
$$


By the ergodic property, for any given constant $m>0$, we have

$$
\lim _{t \rightarrow \infty} \frac{1}{t} \int_{0}^{t}\left(x^{p}(s) \wedge m\right) d s=\int_{R_{+}^{2}}\left(z_{1}^{p} \wedge m\right) \mu\left(d z_{1}, d z_{2}\right), \quad \text { a.s. }
$$

On the other hand, by dominated convergence theorem and (3.20), we get

$$
E\left[\lim _{t \rightarrow \infty} \frac{1}{t} \int_{0}^{t}\left(x^{p}(s) \wedge m\right) d s\right]=\lim _{t \rightarrow \infty} \frac{1}{t} \int_{0}^{t} E\left[x^{p}(s) \wedge m\right] d s \leq K(p)
$$

which together with (3.24) implies

$$
\int_{R_{+}^{2}}\left(z_{1}^{p} \wedge m\right) \mu\left(d z_{1}, d z_{2}\right) \leq K(p)
$$

Letting $m \rightarrow \infty$, we get

$$
\int_{R_{+}^{2}} z_{1}^{p} \mu\left(d z_{1}, d z_{2}\right) \leq K(p)
$$

That is to say, the function $f_{1}(z)=z_{1}^{p}$ is integrable with respect to the measure $\mu$. Therefore, by ergodicity property again, we get

$$
\lim _{t \rightarrow \infty} \frac{1}{t} \int_{0}^{t} x^{p}(s) d s=\int_{R_{+}^{2}} z_{1}^{p} \mu\left(d z_{1}, d z_{2}\right), \quad \text { a.s. }
$$

Besides,

$$
\frac{x(t)-x(0)}{t}=\frac{a}{t} \int_{0}^{t} x(s) d s-\frac{b}{t} \int_{0}^{t} x^{2}(s) d s-\frac{\alpha}{t} \int_{0}^{t} \frac{x(s) y(s)}{1+\beta x(s)} d s+\frac{\sigma_{1}}{t} \int_{0}^{t} x(s) d B_{1}(s) .
$$

Let $M_{1}(t)=\int_{0}^{t} x(s) d B_{1}(s)$ which is a martingale with $M_{1}(0)=0$ and

$$
\limsup _{t \rightarrow \infty} \frac{\left\langle M_{1}, M_{1}\right\rangle_{t}}{t}=\lim _{t \rightarrow \infty} \frac{1}{t} \int_{0}^{t} x_{1}^{2}(s) d s=\int_{R_{+}^{2}} z_{1}^{2} \mu\left(d z_{1}, d z_{2}\right)<\infty,
$$

then by the strong law of large numbers, we get

$$
\lim _{t \rightarrow \infty} \frac{M_{1}(t)}{t}=\lim _{t \rightarrow \infty} \frac{1}{t} \int_{0}^{t} x_{1}(s) d B_{1}(s)=0,
$$


which together with (3.23) and (3.28) implies (3.29) that

$$
\lim _{t \rightarrow \infty} \frac{1}{t} \int_{0}^{t} \frac{x(s) y(s)}{1+\beta x(s)} d s=\frac{a}{\alpha} \int_{R_{+}^{2}} z_{1} \mu\left(d z_{1}, d z_{2}\right)-\frac{b}{\alpha} \int_{R_{+}^{2}} z_{1}^{2} \mu\left(d z_{1}, d z_{2}\right) .
$$

Hence from these arguments, we get the following result.

Theorem 3.3. Assume the same conditions as in Theorem 3.2. Then one has

$$
\begin{gathered}
\lim _{t \rightarrow \infty} \frac{1}{t} \int_{0}^{t} x^{p}(s) d s=\int_{R_{+}^{2}} z_{1}^{p} \mu\left(d z_{1}, d z_{2}\right), \quad \text { a.s., } \\
\lim _{t \rightarrow \infty} \frac{1}{t} \int_{0}^{t} \frac{x(s) y(s)}{1+\beta x(s)} d s=\frac{a}{\alpha} \int_{R_{+}^{2}} z_{1} \mu\left(d z_{1}, d z_{2}\right)-\frac{b}{\alpha} \int_{R_{+}^{2}} z_{1}^{2} \mu\left(d z_{1}, d z_{2}\right) .
\end{gathered}
$$

\section{Extinction}

In this section, we show the situation when system (1.3) will be extinct.

Case 1. $\left(a<\sigma_{1}^{2} / 2\right)$.

Obviously,

$$
d x \leq x(a-b x) d t+\sigma_{1} x d B_{1}(t)
$$

then when $a<\sigma_{1}^{2} / 2$,

$$
\lim _{t \rightarrow \infty} x(t)=0, \quad \text { a.s. }
$$

That is to say, for all $0<\epsilon_{1}<e+\sigma_{2}^{2} / 2$, there exist $T_{1}=T_{1}(\omega)$ and a set $\Omega_{\epsilon_{1}}$ such that $P\left(\Omega_{\epsilon_{1}}\right)>$ $1-\epsilon_{1}$ and $k \alpha x(t) \leq \epsilon_{1}$ for $t \geq T_{1}$ and $\omega \in \Omega_{\epsilon_{1}}$. Then

$$
-e y(t) d t+\sigma_{2} y(t) d B_{2}(t) \leq d y(t) \leq y(t)\left(-e+\epsilon_{1}\right) d t+\sigma_{2} y(t) d B_{2}(t)
$$

and so

$$
\lim _{t \rightarrow \infty} y(t)=0 \text {, a.s. }
$$

Case 2. $\left(a>\sigma_{1}^{2} / 2, e+\sigma_{2}^{2} / 2>k \alpha / \beta\right)$.

Note that

$$
d y(t) \leq y(t)\left(-e+\frac{k \alpha}{\beta}\right) d t+\sigma_{2} y(t) d B_{2}(t)
$$


then if $e+\sigma_{2}^{2} / 2>k \alpha / \beta$, we have

$$
\lim _{t \rightarrow \infty} y(t)=0 \text {, a.s. }
$$

In this situation, for all $0<\epsilon_{2}<a-\sigma_{1}^{2} / 2$, there exist $T_{2}=T_{2}(\omega)$ and a set $\Omega_{\epsilon_{2}}$ such that $P\left(\Omega_{\epsilon_{2}}\right)>1-\epsilon_{2}$ and $\alpha y(t) \leq \epsilon_{2}$ for $t \geq T_{2}$ and $\omega \in \Omega_{\epsilon_{2}}$. Then

$$
\begin{gathered}
x(t)\left(a-b x(t)-\epsilon_{2}\right) d t+\sigma_{1} x(t) d B_{1}(t) \leq d x(t) \leq x(t)(a-b x(t)) d t+\sigma_{1} x(t) d B_{1}(t), \\
\left(a-\frac{\sigma_{1}^{2}}{2}-b x(t)-\epsilon_{2}\right) d t+\sigma_{1} d B_{1}(t) \leq d x(t) \leq\left(a-\frac{\sigma_{1}^{2}}{2}-b x(t)\right) d t+\sigma_{1} d B_{1}(t) .
\end{gathered}
$$

Consider the following equation:

$$
d \Phi(t)=\left(a-\frac{\sigma_{1}^{2}}{2}-b e^{\Phi(t)}\right) d t+\sigma_{1} d B_{1}(t)
$$

If $a>\sigma_{1}^{2} / 2,(4.9)$ has the density $g_{*}(\zeta)$ such that

$$
\frac{1}{2} \sigma_{1}^{2} g_{*}^{\prime}(\zeta)=\left(a_{1}-\frac{\sigma_{1}^{2}}{2}-b_{11} e^{\zeta}\right) g_{*}(\zeta)
$$

Therefore from (4.8) and the arbitrary of $\epsilon_{2}$, we get that the distribution of $\log x(t)$ converges weakly to the probability measure with density $g_{*}$. Thus, from (4.10), we obtain that the distribution of $x(t)$ converges weakly to the probability measure with density $f^{*}(\zeta)=$ $C_{0} \zeta^{2\left(a-\sigma_{1}^{2} / 2\right) / \sigma_{1}^{2}-1} e^{-2 b \zeta / \sigma_{1}^{2}}$, where $C_{0}=\left(2 b / \sigma_{1}^{2}\right)^{2\left(a-\sigma_{1}^{2} / 2\right) / \sigma_{1}^{2}} / \Gamma\left(2\left(a-\sigma_{1}^{2} / 2\right) / \sigma_{1}^{2}\right)$. Besides, from the ergodic theorem and (4.10), it follows that

$$
\lim _{t \rightarrow \infty} \frac{1}{t} \int_{0}^{t} x(s) d s=\int_{-\infty}^{\infty} e^{\zeta} g_{*}(\zeta) d \zeta=\int_{-\infty}^{\infty} \frac{a-\sigma_{1}^{2} / 2}{b} g_{*}(\zeta) d \zeta=\frac{a-\sigma_{1}^{2} / 2}{b} \text { a.s. }
$$

Therefore, by the above arguments, we obtain the following.

Theorem 4.1. Let $(x(t), y(t))$ be the solution of system (1.3) with the initial value $(x(0), y(0)) \in R_{+}^{2}$. Then,

(i) if $a<\sigma_{1}^{2} / 2$, then

$$
\lim _{t \rightarrow \infty} x(t)=0, \quad \lim _{t \rightarrow \infty} y(t)=0, \quad \text { a.s., }
$$


(ii) if $a>\sigma_{1}^{2} / 2, e+\sigma_{2}^{2} / 2>k \alpha / \beta$, then the distribution of $x(t)$ converges weakly to the probability measure with density $f^{*}(\zeta)=C_{0} \zeta^{2\left(a-\sigma_{1}^{2} / 2\right) / \sigma_{1}^{2}-1} e^{-2 b \zeta / \sigma_{1}^{2}}$, where $C_{0}=$ $\left(2 b / \sigma_{1}^{2}\right)^{2\left(a-\sigma_{1}^{2} / 2\right) / \sigma_{1}^{2}} / \Gamma\left(2\left(a-\sigma_{1}^{2} / 2\right) / \sigma_{1}^{2}\right)$, and

$$
\lim _{t \rightarrow \infty} \frac{1}{t} \int_{0}^{t} x(s) d s=\frac{a-\sigma_{1}^{2} / 2}{b}, \quad \lim _{t \rightarrow \infty} y(t)=0, \quad \text { a.s. }
$$

\section{Appendix}

For the completeness of the paper, in this section, we list some theories about stationary distribution (see [15]).

Let $X(t)$ be a homogeneous Markov process in $E_{l}$ ( $E_{l}$ denotes euclidean $l$-space) described by

$$
d X(t)=b(X) d t+\sum_{r=1}^{k} g_{r}(X) d B_{r}(t)
$$

The diffusion matrix is $A(x)=\left(a_{i j}(x)\right), a_{i j}(x)=\sum_{r=1}^{k} g_{r}^{i}(x) g_{r}^{j}(x)$.

Assumption $B$. There exists a bounded domain $U \subset E_{l}$ with regular boundary $\Gamma$, having the following properties.

(B.1) In the domain $U$ and some neighbourhood thereof, the smallest eigenvalue of the diffusion matrix $A(x)$ is bounded away from zero.

(B.2) If $x \in E_{l} \backslash U$, the mean time $\tau$ at which a path issuing from $x$ reaches the set $U$ is finite, and $\sup _{x \in K} E_{x} \tau<\infty$ for every compact subset $K \subset E_{l}$.

Lemma A.1 (see [15]). If (B) holds, then the Markov process $X(t)$ has a stationary distribution $\mu(\cdot)$. Let $f(\cdot)$ be a function integrable with respect to the measure $\mu$. Then $P_{x}\left\{\lim _{T \rightarrow \infty} 1 / T \int_{0}^{T} f(X(t)) d t=\right.$ $\left.\int_{E_{l}} f(x) \mu(d x)\right\}=1$ for all $x \in E_{l}$.

Remark A.2. The proof is given in [15]. Exactly, the existence of stationary distribution with density is referred to Theorem 4.1, Page 119, and Lemma 9.4, Page 138, in [4]. The weak convergence and the ergodicity is obtained in Theorem 5.1, Page 121, and Theorem 7.1, Page 130 , in [4].

To validate (B.1), it suffices to prove that $F$ is uniformly elliptical in any bounded domain $D$, where $F u=b(x) \cdot u_{x}+(1 / 2) \operatorname{tr}\left(A(x) u_{x x}\right)$; that is, there is a positive number $M$ such that $\sum_{i, j=1}^{k} a_{i j}(x) \xi_{i} \xi_{j} \geq M|\xi|^{2}, x \in \bar{D}, \xi \in R^{k}$ (see Chapter 3, Page 103 of [19] and Rayleigh's principle in [20, Chapter 6, Page 349]). To verify (B.2), it is sufficient to show that there exists some neighborhood $U$ and a nonnegative $C^{2}$-function such that and for any $E_{l} \backslash U, L V$ is negative (for details refer to [21, Page 1163]).

Lemma A.3. Let $X(t)$ be a regular temporally homogeneous Markov process in $E_{l}$. If $X(t)$ is recurrent relative to some bounded domain $U$, then it is recurrent relative to any nonempty domain in $E_{l}$. 


\section{Acknowledgments}

The work was supported by the Ministry of Education of China (no. 109051), the Ph.D. Programs Foundation of Ministry of China (no. 200918), NSFC of China (no. 10971021), and the Graduate Innovative Research Project of NENU (no. 09SSXT117), Youth Fund of Jiangsu Province (no. BK2012208), and the Tian Yuan Special Funds of the National Natural Science Foundation of China (no. 11226205).

\section{References}

[1] H. I. Freedman, Deterministic Mathematical Models in Population Ecology, Marcel Dekker, New York, NY, USA, 1980.

[2] C.S. Holling, "The components of predation as revealed by a study of small-mammal predation of the european pine sawy," Canadian Entomologist, vol. 91, pp. 293-320, 1959.

[3] N. G. Hairston, F. E. Smith, and L. B. Slobodkin, "Community structure, population control and competition," The American Naturalist, vol. 94, pp. 421-425, 1960.

[4] M. L. Rosenzweig, "Paradox of enrichment: destabilization of exploitation ecosystems in ecological time," Science, vol. 171, pp. 385-387, 1969.

[5] L. S. Chen and Z. J. Jing, "Existence and uniqueness of limit cycles for the differential equations of predator-prey interaction," Chinese Science Bulletin, vol. 29, no. 9, pp. 521-523, 1984.

[6] X. N. Liu and L.S. Chen, "Complex dynamics of Holling type II Lotka-Volterra predator-prey system with impulsive perturbations on the predator," Chaos, Solitons and Fractals, vol. 16, no. 2, pp. 311-320, 2003.

[7] S. W. Zhang and L. S. Chen, "A Holling II functional response food chain model with impulsive perturbations," Chaos, Solitons and Fractals, vol. 24, no. 5, pp. 1269-1278, 2005.

[8] S. W. Zhang, D. J. Tan, and L. S. Chen, "Chaos in periodically forced Holling type II predator-prey system with impulsive perturbations," Chaos, Solitons and Fractals, vol. 28, no. 2, pp. 367-376, 2006.

[9] R. M. May, Stability and Complexity in Model Ecosystems, Princeton University Press, New Jersey, NJ, USA, 1973.

[10] C. Ji, D. Jiang, and N. Shi, "Analysis of a predator-prey model with modified Leslie-Gower and Holling-type II schemes with stochastic perturbation," Journal of Mathematical Analysis and Applications, vol. 359, no. 2, pp. 482-498, 2009.

[11] C. Y. Ji, D. Q. Jiang, and X. Y. Li, “Qualitative analysis of a stochastic ratio-dependent predator-prey system," Journal of Computational and Applied Mathematics, vol. 235, no. 5, pp. 1326-1341, 2011.

[12] C. Y. Ji, D. Q. Jiang, and N. Z. Shi, "A note on a predator-prey model with modified Leslie-Gower and Holling-type II schemes with stochastic perturbation," Journal of Mathematical Analysis and Applications, vol. 377, no. 1, pp. 435-440, 2011.

[13] C. Y. Ji and D. Q. Jiang, "Dynamics of a stochastic density dependent predator-prey system with Beddington-DeAngelis functional response," Journal of Mathematical Analysis and Applications, vol. 381, no. 1, pp. 441-453, 2011.

[14] J. L. Lv and K. Wang, "Asymptotic properties of a stochastic predator-prey system with Holling II functional response," Communications in Nonlinear Science and Numerical Simulation, vol. 16, no. 10, pp. 4037-4048, 2011.

[15] R. Z. Has'meminskii, Stochastic Stability of Differential Equations, vol. 7, Sijthoff \& Noordhoff, Alphen aan den Rijn, The Netherlands, 1980.

[16] R. Rudnicki, "Long-time behaviour of a stochastic prey-predator model," Stochastic Processes and their Applications, vol. 108, no. 1, pp. 93-107, 2003.

[17] R. Rudnicki and K. Pichorr, "Influence of stochastic perturbation on prey-predator systems," Mathematical Biosciences, vol. 206, no. 1, pp. 108-119, 2007.

[18] X. R. Mao, Stochastic Differential Equations and Applications, Horwood, Chichester, UK, 1997.

[19] T. C. Gard, Introduction to STochastic Differential Equations, vol. 270, Madison Avenue, New York, NY, USA, 1988.

[20] G. Strang, Linear Algebra and Its Applications, Thomson Learning, 1988.

[21] C. Zhu and G. Yin, "Asymptotic properties of hybrid diffusion systems," SIAM Journal on Control and Optimization, vol. 46, no. 4, pp. 1155-1179, 2007. 


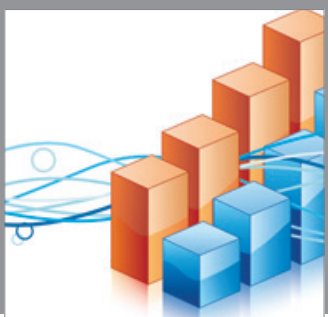

Advances in

Operations Research

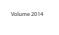

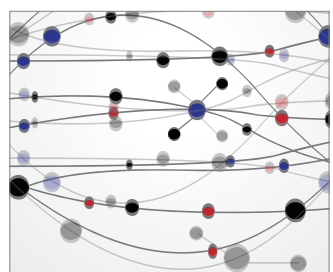

\section{The Scientific} World Journal
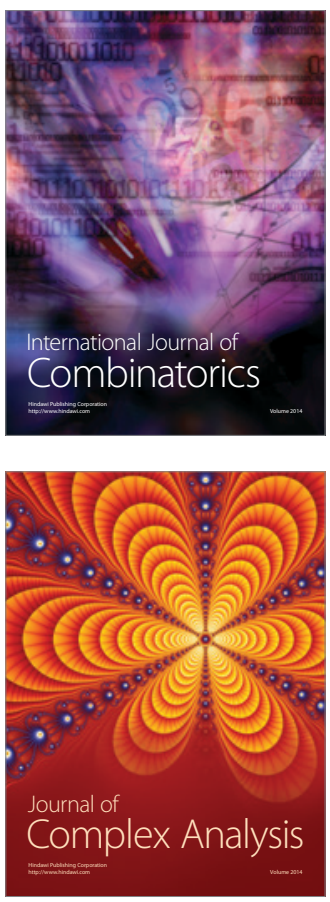

International Journal of

Mathematics and

Mathematical

Sciences
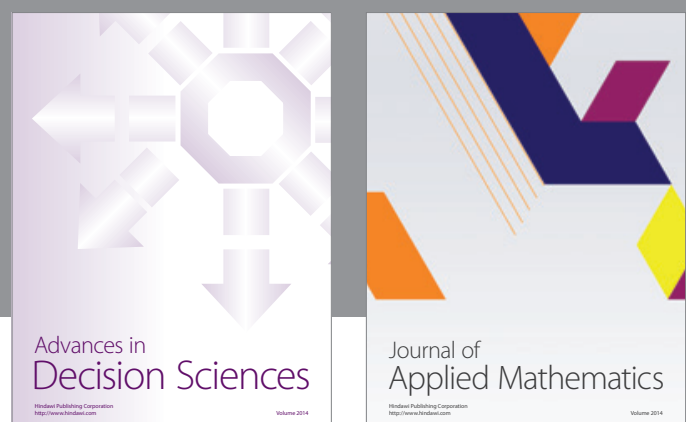

Journal of

Applied Mathematics
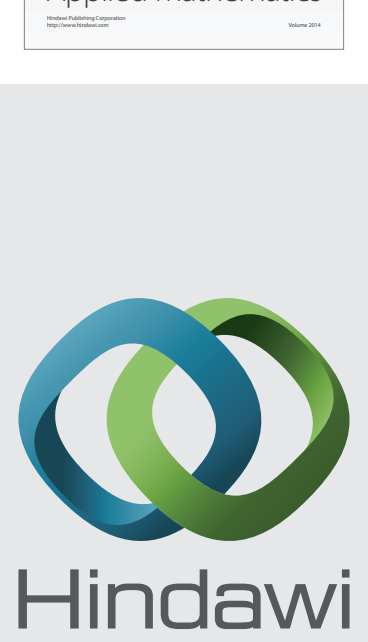

Submit your manuscripts at http://www.hindawi.com
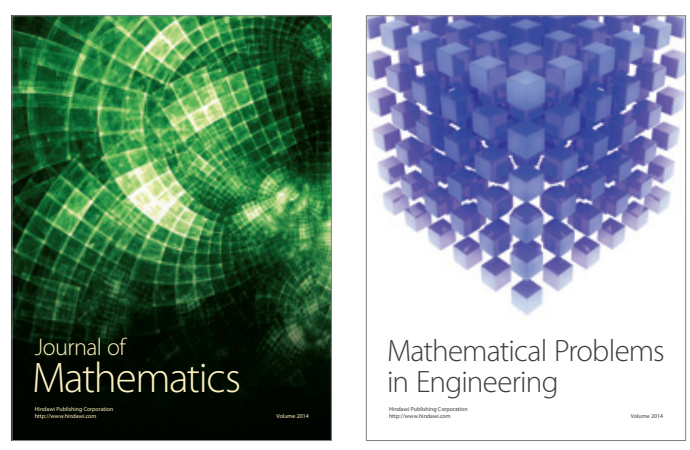

Mathematical Problems in Engineering
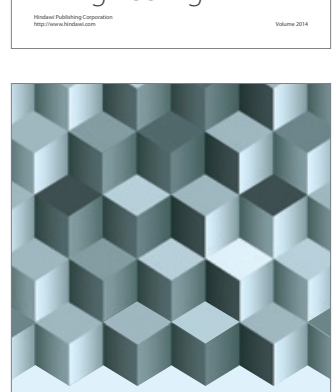

Journal of

Function Spaces
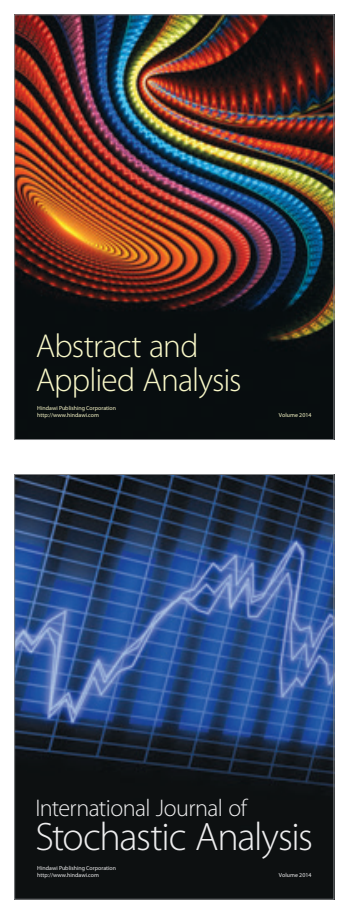

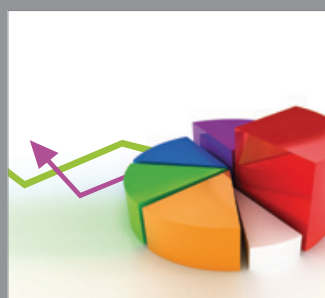

ournal of

Probability and Statistics

Promensencen
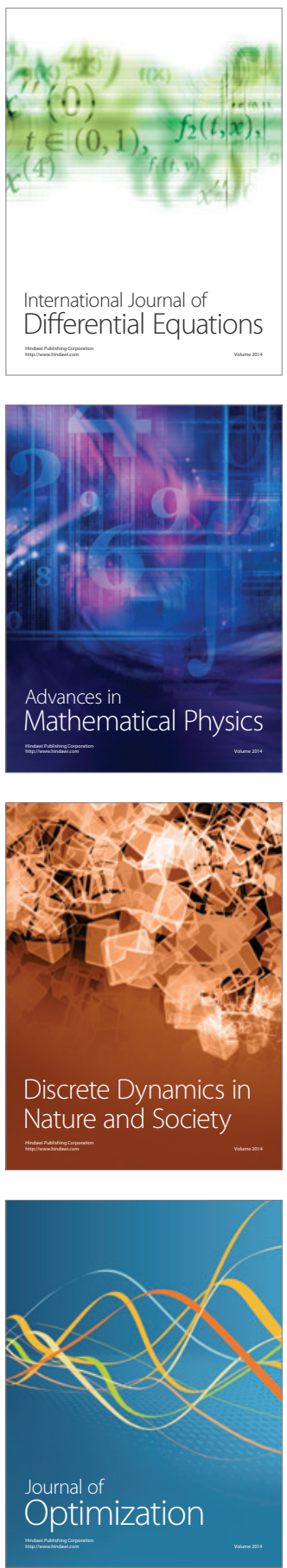